\title{
INTRINSIC DEFECTS IN NEUTRON-IRRADIATED SILICON AN INFRARED STUDY
}

\author{
F. CARTON-MERLET $(*)$, B. PAJOT $(*)$ \\ Laboratoire d'Infrarouge, Bât. 350, Université Paris XI, 91405 Orsay, France \\ and \\ P. VAJDA
}

Equipe de Recherche du C.N.R.S., Laboratoire de Chimie Physique, Bât. 350, Université Paris XI, 91405 Orsay, France

(Reçu le 20 janvier 1978, accepté le 2 mars 1978)

\begin{abstract}
Résumé. - On montre que la présence ou l'absence des bandes d'absorption observées à basse température entre 3,3 et 3,6 $\mu \mathrm{m}$ dans le silicium irradié peut être contrôlée par illumination de l'échantillon avec du rayonnement infrarouge d'énergie adéquate. Ceci correspond à la capture d'un photoélectron ou à la photo-éjection d'un électron par le défaut concerné, et l'on discute de l'état de charge optiquement actif du défaut. On rapporte aussi l'observation de nouvelles raies d'absorption IR dues à des défauts intrinsèques dont on discute brièvement la nature en fonction des résultats obtenus après recuit.
\end{abstract}

Abstract. - We show that the low temperature observation of the near IR absorption bands at 3.3-3.6 $\mu \mathrm{m}$ in irradiated silicon can be monitored by illumination of the sample by infrared radiation of selected energies. This behaviour is ascribed to the capture of a photoelectron or to its photorelease by the defect involved, and we discuss its IR active charge state. We also report the observation of new IR absorption lines prior to annealing, due to intrinsic defects whose nature is briefly discussed in relation to the annealing results.

1. Introduction. - One of the problems encountered in the study of the near infrared (IR) absorption of irradiated silicon is the relation between the observation of a given band and the position of the Fermi level, which is one of the parameters determining the charge state of the defects at low temperature [1-3]. The identification of the charge states of radiation defects associated with IR bands has been left open in many cases, even with the help of EPR results.

In this letter, we give evidence that the group of absorption bands in the vicinity of $3.3 \mu \mathrm{m}$ (bands at $3.30,3.46$ and $3.62 \mu \mathrm{m}$ ) is due to a defect - very likely the divacancy $[4,5]$ - whose charge state is not IR active at thermal equilibrium at $8 \mathrm{~K}$ in the neutronirradiated samples investigated in the present study. We report also the observation of new sharp absorption lines near $3.6 \mu \mathrm{m}$, which exhibit the same photosensitivity as the $3.3 \mu \mathrm{m}$ group.

(*) Present address : Groupe de Physique des Solides de l'E.N.S., Tour 23, 2 place Jussieu, 75221 Paris Cedex 05, France.
This work was initiated by the accidental observation of the quenching of the $3.3 \mu \mathrm{m}$ bands when the white source of the spectrometer was filtered with a germanium disk $[6,7]$.

2. Experimental conditions. - The two extreme samples used for this investigation were a p-type (B-doped) sample with $N_{\mathrm{a}}-N_{\mathrm{d}}=1.3 \times 10^{17} \mathrm{~cm}^{-3}$ and a n-type (P-doped) sample with

$$
N_{\mathrm{d}}-N_{\mathrm{a}}=3 \times 10^{16} \mathrm{~cm}^{-3}
$$

both cut from FZ crystals. The oxygen concentration for the B-doped sample was less than $3 \times 10^{13} \mathrm{at} / \mathrm{cm}^{3}$. The carbon concentration $\left({ }^{1}\right)$ has not been measured. The fast neutron dose was $1.8 \times 10^{17} \mathrm{~cm}^{-2}$ for the p-type sample and $9 \times 10^{16} \mathrm{~cm}^{-2}$ for the n-type

( $\left.{ }^{1}\right)$ The limit of sensitivity for oxygen detection in FZ silicon crystals has been discussed in Ref. [18]. Oxygen was not detected by low-temperature IR absorption in this sample. 
sample. The phosphorus concentration added by the transmutation of silicon by thermal neutrons capture is below $2 \times 10^{14} \mathrm{at} / \mathrm{cm}^{3}$. After irradiation [7], both samples were intrinsic. The isochronal annealing was performed in $20 \mathrm{~min}$. steps of $\Delta T=50 \mathrm{~K}$ from room temperature up to $548 \mathrm{~K}$.

A monochromator fitted with a 300 lines $/ \mathrm{mm}$ grating blazed at $3 \mu \mathrm{m}$ was used and the grating orders higher than the first one were eliminated using a long wavelength filter before the monochromator. The monochromatic beam was focussed on the sample placed in a liquid helium optical cryostat. The tail of the cryostat has two pairs of perpendicular apertures. One of the apertures not used for the transmission is fitted with a sodium chloride window. This allows illumination of the sample perpendicularly to the direction of the analysing beam. It is realized with a quartz-halogen lamp, adequate focussing optics and filters. This auxiliary system is light-tight so that no white light can reach the sample unless deliberately admitted. The role of the filters is to monitor the creation rate of electron-hole pairs and the ionization rate of the traps.

3. Experimental results. - The first series of experiments consisted in a repetition of those reported in reference [7] with the difference that instead of using a commercial spectrometer (cryostat placed before the monochromator) we used the above-described set-up. This latter design is compulsory when illuminating with light of an energy smaller than that used for the analysis and it does not modify the intensity of the analysing beam. The first experiments were conducted in total darkness after the filling of the cryostat with liquid helium. The results were identical for both samples and quite similar to those reported previously. We noted that the most efficient bleaching of the $3.62 \mu \mathrm{m}$ band was obtained using a $2.3 \mu \mathrm{m}$ long-pass filter, as shown in figure 1 . After an equilibrium had been reached, perpendicular illumination was found to reduce the absorption coefficient of the $3.62 \mu \mathrm{m}$ band by about a factor of $100\left({ }^{2}\right)$. The same effect was also observed for the bands at 3.30 and $3.46 \mu \mathrm{m}$.

The fact that, once bleached, the band did not reappear when the illumination was stopped made us think of a trapping effect, easier to understand than a quenching effect. Consequently the cryostat was blinded before cooling the sample below room temperature. Under this condition and operating in total darkness, the $3.3 \mu \mathrm{m}$ bands went undetected for both samples at $8 \mathrm{~K}$. The monochromator was also scanned in the $3.9 \mu \mathrm{m}$ region, but no absorption band was detected. When ambient incandescent light was switched on, the $3.3 \mu \mathrm{m}$ bands reappeared, indicating

$\left(^{2}\right)$ With white light illumination the absorption coefficient of the n-type and p-type samples at $8 \mathrm{~K}$ were 5.2 and $3.5 \mathrm{~cm}^{-1}$ respectively, before annealing.

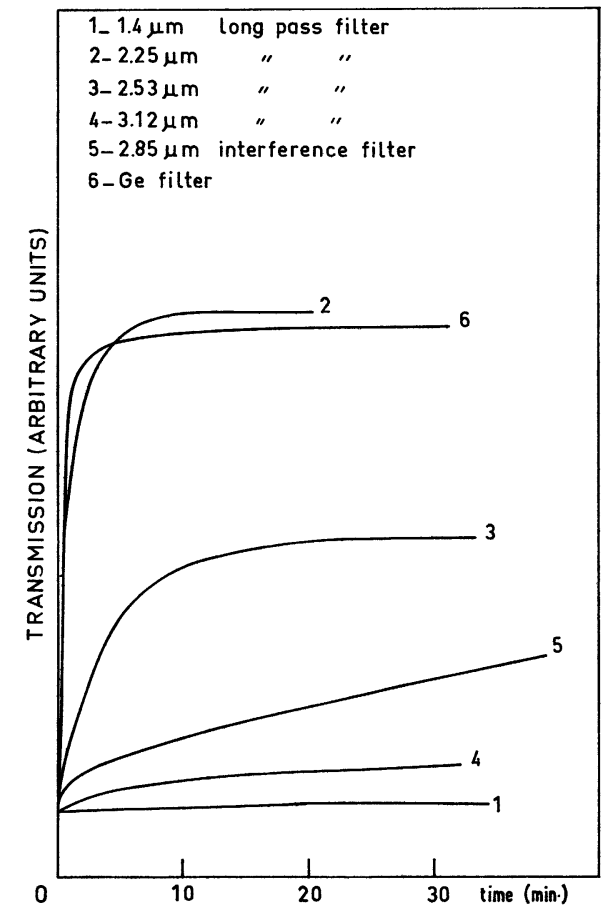

FIG. 1. - Decrease with time of the peak absorption of the $3.62 \mu \mathrm{m}$ band (p-type sample), recorded with monochromatic radiation, under the influence of perpendicular illumination with filtered IR radiation. Prior to each measurement, the centre is repopulated with white light. The spontaneous decay curve (not shown) follows curve 4 .

that the radiation defect responsible for the bands was not in the appropriate charge state at darkness equilibrium for IR absorption. The IR active charge state could be reached by trapping of an electron (or of a hole) from a photogenerated pair. Under this assumption, we can investigate the energy dependence of the formation of an electron-hole pair by merely determining the energy of the perpendicular radiation corresponding to the threshold for the observation of the $3.62 \mu \mathrm{m}$ band.

The trapping efficiency obtained with ambient incandescent light was slightly lower than that obtained using unfiltered perpendicular pumping, but both results were reproducible. When the excitation with ambient light and/or with the unfiltered quartz-halogen lamp was stopped, a small decrease with time in the absorption coefficient of the band was observed, and it is with this spontaneous decay that the light-induced decay or increase must be compared.

The same phenomena were also observed with the sample held at $77 \mathrm{~K}$.

We have tried to investigate the role of the $1.7 \mu \mathrm{m}$ band by selective illumination at this wavelength using an interference filter, but no definite conclusion can be drawn now from our results.

Besides the $3.3 \mu \mathrm{m}$ bands we have also observed weak lines at lower energy (Fig. 2). They have been seen in both samples and also in an Al-doped sample at liquid helium temperature, but they are barely detectable at $77 \mathrm{~K}$. In contrast with the $3.62 \mu \mathrm{m}$ band, 


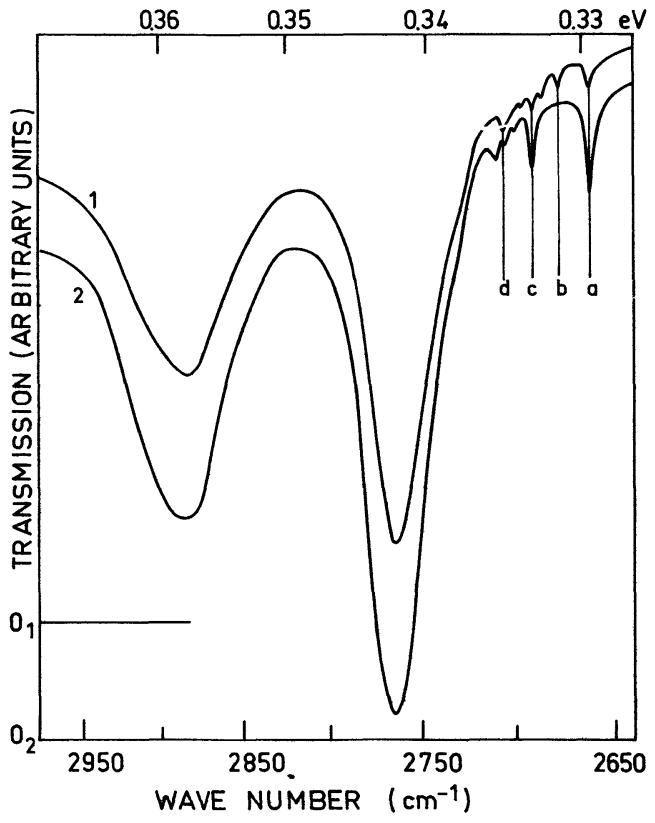

FIG. 2. - Typical transmission spectra $(T \sim 8 \mathrm{~K})$ of the two irradiated Si samples mentioned in the text 1) n-type $d=0.4 \mathrm{~cm}$ annealed at room temperature ; 2) p-type $d=0.46 \mathrm{~cm}$ annealed at $448 \mathrm{~K}$. A low energy shoulder of the strong band at $2766 \mathrm{~cm}^{-1}$ $(3.62 \mu \mathrm{m})$ can be seen in both spectra. This shoulder anneals out at $498 \mathrm{~K}$. The letters refer to the annealing curves.

\section{TABLE I}

Spectral characteristics of the new intrinsic lines observed in neutron-irradiated silicon at $8 \mathrm{~K}$

\begin{tabular}{|c|c|c|c|c|}
\hline \multicolumn{2}{|c|}{$\begin{array}{l}\text { Position of the } \\
\text { line }\left({ }^{a}\right)\end{array}$} & \multicolumn{2}{|c|}{$\begin{array}{c}\text { Maximum absorption } \\
\text { coefficient }\end{array}$} & \multirow{2}{*}{$\begin{array}{c}\text { Disappears } \\
\text { at }\end{array}$} \\
\hline $\mathrm{cm}^{-1}$ & $\mathrm{meV}$ & $\mathrm{cm}^{-1}$ & K & \\
\hline - & - & - & - & - \\
\hline 2708.2 & 335.82 & 0.07 & 448 & 498 \\
\hline $2703.5\left(^{b}\right)$ & 335.23 & 0.09 & 298 & 348 \\
\hline 2689.3 & 333.47 & 0.18 & 448 & 498 \\
\hline 3684.1 & 332.83 & 0.02 & 298 & 448 \\
\hline 2675.9 & 331.81 & 0.03 & 348 & 398 \\
\hline 2659.9 & 329.83 & 0.31 & 448 & 498 \\
\hline $2631.0\left({ }^{b}\right)$ & 326.24 & 0.01 & $448\left(^{c}\right)$ & \\
\hline $2627.4\left(^{d}\right)$ & 325.80 & 0.04 & $498\left(^{(c)}\right.$ & \\
\hline
\end{tabular}

$\left({ }^{a}\right)$ Accuracy : $\pm 0.3 \mathrm{~cm}^{-1}$ unless otherwise specified.

(b) Accuracy : $\pm 0.6 \mathrm{~cm}^{-1}$.

$\left({ }^{c}\right)$ Observed only at the temperature indicated (annealing steps of $50 \mathrm{~K}$ ).

( $\left.{ }^{d}\right)$ Line 7 of reference [7] at $3.80 \mu \mathrm{m}$.

whose width is $\sim 2 \mathrm{meV}$ at $8 \mathrm{~K}$, these lines have a width $\sim 0.3 \mathrm{meV}$ at this temperature. Their energy positions are given in table 1 and a rough idea of their annealing behaviour in figure 3 . After annealing at $225^{\circ} \mathrm{C}$, only one line is observed at $325.8 \mathrm{meV}$. This line was also reported for $\mathrm{Li}$-doped $\mathrm{Si}$ in reference [7] (line 7 at $3.80 \mu \mathrm{m}$ ) after a $200^{\circ} \mathrm{C}$ anneal. Hence, line 7 of the above reference is not due to a Li-defect complex. From the annealing behaviour of this group of lines, it seems that at least three distinct radiation defects are involved. As to their origin we rule out impurity defect complexes involving either electrically

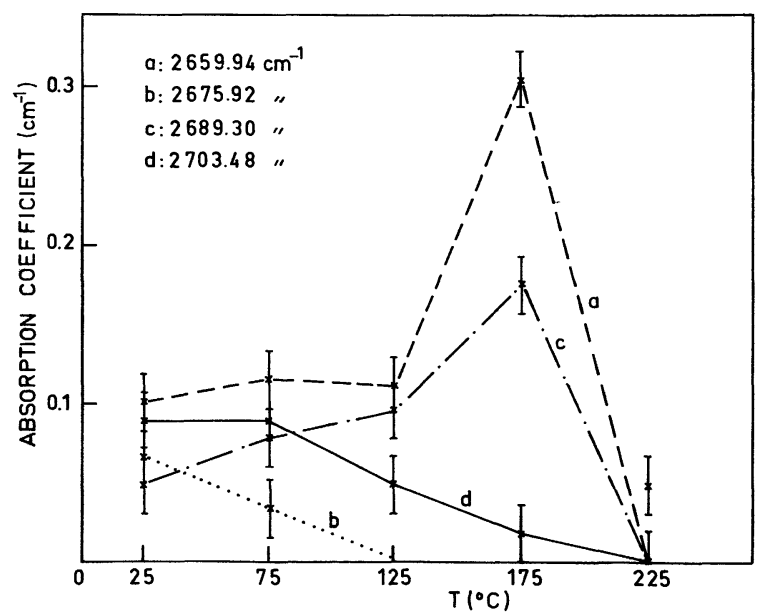

FIG. 3. - Annealing curves of the most intense of the new intrinsic lines.

active or neutral impurities as concentration variations over several orders of magnitude do not change their relative intensities with respect to the $3.62 \mu \mathrm{m}$ band. These new lines show the same phototrapping effect as the $3.3 \mu \mathrm{m}$ bands, but we cannot tell now if the related defects trap the same carrier as the divacancy or the carrier of the opposite charge.

4. Discussion. - Let us first consider the $3.3 \mu \mathrm{m}$ bands. They are conveniently labelled by their wavelength position as the $3.62,3.46$ and $3.30 \mu \mathrm{m}$ bands. (Lines 6, 5 and 4, respectively of reference [7].) There are also weaker bands at higher energies, but they are not considered here. The 3.62 and $3.46 \mu \mathrm{m}$ bands show the same annealing behaviour and their bleaching by infrared radiation show the same spectral dependence; moreover their stress induced splitting and dichroism are very similar [5]. For these reasons, we are inclined to ascribe them to the same defect in the same charge state. Owing to its weak intensity, the $3.30 \mu \mathrm{m}$ band has not been thoroughly investigated but we have found that its bleaching properties are similar to those of its two companions. Lappo and Tkachev [8] have attributed the 3.46 and $3.30 \mu \mathrm{m}$ broad bands to phonon replicas of the relatively sharp $3.62 \mu \mathrm{m}$ band and this does not look unrealistic (see table II).

\section{TABLE II}

Position of the " $3.3 \mu \mathrm{m}$ » bands and comparison of the higher energy bands with phonon replicas of the $2766 \mathrm{~cm}^{-1}$ band. The phonon assignment is indicated in parenthesis $\left({ }^{a}\right)$.

\begin{tabular}{cccc}
$\begin{array}{c}\text { Band label } \\
\mu \mathrm{m}\end{array}$ & \multicolumn{2}{c}{ Position } & Calculated position \\
- & $\mathrm{cm}^{-1}$ & $\mathrm{meV}$ & $\mathrm{cm}^{-1}$ \\
3.62 & $2766 \pm 2$ & 343.0 & - \\
3.46 & $2890 \pm 3$ & 358.4 & $2880(\mathrm{TA}(\mathrm{L}))$ \\
3.30 & $3027 \pm 3$ & 375.3 & $3029(\mathrm{TA}(\mathrm{L})+\mathrm{TA}(\mathrm{X}))$
\end{tabular}

$\left({ }^{a}\right)$ Reference [9] 
We wish now to make some guesses concerning the defect associated with these bands and to point out their consequences. We suppose first that it is the negative divacancy $\left(\mathrm{V}_{2}^{-}\right)$. This defect gives rise to the Si G7 EPR spectrum [4], so that IR and EPR spectra should be correlated. The absence of the IR bands in the n-type sample at equilibrium in darkness could be due to the fact that all the divacancies have turned into the zero charge state, i.e. the fast neutron fluence of $9 \times 10^{16} \mathrm{~cm}^{-2}$ causes the Fermi level to recede deeper than $E_{\mathrm{c}}-0.4 \mathrm{eV}$ (the ground state energy [10] of $\mathrm{V}_{2}^{-}$) in a n-type sample with $n=3 \times 10^{16} \mathrm{~cm}^{-3}$. White light illumination would cause the trapping of photogenerated electrons by $V_{2}^{0}$ and the trapping of the photogenerated hole by a defect with a higher hole capture-cross-section than $\mathrm{V}_{2}^{0}$ or $\mathrm{V}_{2}^{-}\left({ }^{3}\right)$. We have then to interpret the bleaching of the IR bands under illumination with photons of energy $c a 0.55 \mathrm{eV}(2.3 \mu \mathrm{m})$ either as the direct ionization of $\mathrm{V}_{2}^{-}$into the conduction band or as the ionization of another defect in the valence band, followed by the recombination of the free hole with $\mathrm{V}_{2}^{-}$(indirect process). This photobleaching must be compared with the intensity decrease under IR illumination of the Si G7 and Si A10 EPR spectra observed at $77 \mathrm{~K}$ by Lee et al. [12]. These authors observe a low-energy threshold of about $0.4 \mathrm{eV}$ for the decrease of the EPR spectra, that they correlate with the ionization energy reported for $\mathrm{V}_{2}^{-}$. This is substantiated by the fact that the photoionization cross-section for deep levels in silicon is maximum at energies notably higher than the ionization energy [13]. The photoionization cross-section of $\mathrm{Zn}$ in $\mathrm{Si}$ peaks at $0.5 \mathrm{eV}$, whereas the thermal ionization energy is $\sim 0.3 \mathrm{eV}$; at this latter energy, the cross-section is nearly two orders of magnitude lower than the maximum value [14]. We can use this argument to attribute the IR bands to $V_{2}^{-}$with some conditions on the electron and hole capture cross section for the various charge states of $\mathrm{V}_{2}$. The crucial test remains however the correlation of the EPR and IR spectra in low resistivity n-type samples irradiated with lower fluences $\left(\sim 10^{16} \mathrm{~cm}^{-2}\right)$. In these samples, the IR photobleaching of the $3.3 \mu \mathrm{m}$ bands must be less pronounced or even inexistent as it depends on the position of the Fermi level; this is a possible explanation for the results obtained by Chen and Corelli [5].

With the above attribution, the excited state of $\mathrm{V}_{2}^{-}$ corresponding to the $3.62 \mu \mathrm{m}$ band $(0.343 \mathrm{eV})$ lies $60 \mathrm{meV}$ below the conduction band: it can then be thermalised at moderate temperature $(\sim 100 \mathrm{~K})$, but no such peak has been reported in the photoconductivity spectrum.

The attribution of the $3.3 \mu \mathrm{m}$ bands to $\mathrm{V}_{2}^{=}$or to $\mathrm{V}_{2}^{+}$ does not seem realistic considering ground state energies of $E_{\mathrm{c}}-0.23 \mathrm{eV}$ and $E_{\mathrm{v}}+0.25 \mathrm{eV}$, respectively. The attribution to $\mathrm{V}_{2}^{0}\left(E_{\mathrm{c}}-0.55 \mathrm{eV}\right)$ would fit the wavelength of $2.3 \mu \mathrm{m}$ but it is difficult to understand from our results as it implies that :

a) no neutral divacancies exist in the samples investigated at equilibrium in darkness;

b) the same IR photobleaching is observed for both $V_{2}^{0}$ (IR) and $V_{2}^{-}$(EPR).

As a first step towards the understanding of the new absorption bands, we remark that most of them are observed prior to annealing above room temperature. The only intrinsic defects which have been observed in neutron-irradiated $\mathrm{Si}$ annealed at room temperature are the planar tetravacancy (Si P3), the positively charged di-interstitial (Si P6) and the Si A5 center [15]. The lines at 335.8, 335.2, 333.5, 332.8 and $329.8 \mathrm{meV}$ should then be related to one of these defects but the annealing steps and the intensity of the lines prevent more accurate predictions. The line at $325.8 \mathrm{meV}$ is observed in a restricted domain of temperature and it could be correlated with the Si A2 center [16]. Because of their low intensity and of the annealing steps chosen, no correlation can be made for the lines at 331.8 and $326.2 \mathrm{meV}$. One of the critical points in the study of neutron irradiated silicon is the relative importance of the defects in the damage cluster with respect to the defects outside the cluster [11] and this can be a possible limitation for significant intercomparisons between IR and EPR measurements.

An important point to keep in mind for the EPR comparisons is that all these lines behave in a way comparable to that at $3.3 \mu \mathrm{m}$. The next step, which is the determination of the symmetry of the defects, their ionization energy and their IR active charge state is presently being investigated.

Acknowledgments. - We whish to thank the Société Vieille Montagne for kindly providing one of the crystals used for this study. The help of Mrs. A. Bessis and Y. Bismuth during the neutron irradiation and the technical assistance of Mr. A. M. Jean Louis are gratefully acknowledged.

\section{References}

[1] Fan, H. Y. and Ramdas, A. K., J. Appl. Phys. 30 (1959) 1127. [2] Cheng, L. J., Corelli, J. C., Corbett, J. W. and Watkins, G. D., Phvs. Rev. 152 (1966) 761.

$\left({ }^{3}\right)$ For neutron fluences $\left(7 \times 10^{15} \mathrm{~cm}^{-2}\right)$ the Si G7 spectrum has been observed by Daly and Noffke [11] in a FZ sample with $n=6 \times 10^{16} \mathrm{~cm}^{-3}$ cooled from room temperature in darkness but not in a FZ sample with $n=1 \times 10^{16} \mathrm{~cm}^{-3}$ under the same conditions.
[3] Lotkova, E. N., Proc. P.N. Lebedev Physics Institute 37, Ed. Ac. D. V. Skobel'tsyn (Electrical and Optical Properties of Semiconductors, Consultant Bureau, New York, 1968) p. 87.

[4] Watkins, G. D. and Corbett, J. W., Phys. Rev. 138 (1972) A 543.

[5] Chen, C. S. and Corelli, J. C., Phys. Rev. B 5 (1972) 1505.

[6] Bean, A. R., Newman, R. C. and Smith, R. S., J. Phys. Chem. Solids 31 (1970) 739. 
[7] Merlet, F., Pajot, B. and Vajda, P., J. Appl. Phys. 47 (1976) 1729.

[8] Lappo, M. T. and Tkachev, V. D., Sov. Phys. Semicond. 4 (1971) 1882.

[9] Birman, J. L., Encyclopedia of Physics, edited by L. Genzel (Springer Verlag, Berlin, New York) 1974, XXV/2c, p. 411.

[10] Evwaraye, A. O. and Sun, E., J. Appl. Phys. 47 (1976) 3776.

[11] DALY, D. F. and NoFFKe, H. E., Radiat. Eff. 8 (1971) 203

[12] LeE, H. Y., Bilash, T. D. and Corbett, J. W., Radiat. Eff. 29 (1976) 7 .
[13] Pantelides, S. and Bernholc, J., Radiat. Eff. in Semiconductors, 1976 (Inst. Phys. Conf. Ser. 31) p. 465.

[14] Sclar, N., Infrared Phys. 16 (1976) 435.

[15] Lee, Y. H., Gerasimenko, N. N. and Corbett, J. W., Phys. Rev. B 14 (1976) 4506 and references therein.

[16] LeE, Y. H., KIM, M. and CoRbeTt, J. W., Radiat. Eff. 15 (1972)

[17] Daly, D. F. and NoffKe, H. E., Radiat. Eff. 10 (1971) 191.

[18] Pajot, B., Analysis 5 (1977) 293. 